\title{
Addressing Household Food Insecurity in Canada - Position Statement and Recommendations - Dietitians of Canada
}

\section{POSITION STATEMENT}

It is the position of Dietitians of Canada that household food insecurity is a serious public health issue with profound effects on physical and mental health and social well-being. All households in Canada must have sufficient income for secure access to nutritious food after paying for other basic necessities.

Given the alarming prevalence, severity and impact of household food insecurity in Canada, Dietitians of Canada calls for a pan-Canadian, government-led strategy to specifically reduce food insecurity at the household level, including policies that address the unique challenges of household food insecurity among Indigenous Peoples. Regular monitoring of the prevalence and severity of household food insecurity across all of Canada is required. Research must continue to address gaps in knowledge about household vulnerability to food insecurity and to evaluate the impact of policies developed to eliminate household food insecurity in Canada.

\section{Dietitians of Canada recommends:}

1. Development and implementation of a pan-Canadian governmentled strategy that includes coordinated policies and programs, to ensure all households have consistent and sufficient income to be able to pay for basic needs, including food.

2. Implementation of a federally-supported strategy to comprehensively address the additional and unique challenges related to household food insecurity among Indigenous Peoples, including assurance of food sovereignty, with access to lands and resources, for acquiring traditional/country foods, as well as improved access to more affordable and healthy store-bought/market foods in First Nation reserves and northern and remote communities.

3. Commitment to mandatory, annual monitoring and reporting of the prevalence of marginal, moderate and severe household food insecurity in each province and territory across Canada, including among vulnerable populations, as well as regular evaluation of the impact of poverty reduction and protocols for screening within the health care system.

4. Support for continued research to address gaps in knowledge about populations experiencing greater prevalence and severity of household food insecurity and to inform the implementation and evaluation of strategies and policies that will eliminate household food insecurity in Canada.

(Can J Diet Pract Res. 2016;77:159)

(DOI: 10.3148/cjdpr-2016-019)

Published at dcjournal.ca on 19 August 2016.

\section{ÉNONCÉ DE POSITION}

Les diététistes du Canada sont d'avis que l'insécurité alimentaire des ménages est un enjeu de santé publique sérieux ayant d'importants effets sur la santé physique et mentale de même que sur le bien-être social. Tous les ménages au Canada doivent disposer d'un revenu suffisant pour avoir un accès adéquat à des aliments nutritifs après avoir payé les autres nécessités de base.

En raison de la prévalence, de la gravité et de l'impact alarmants de l'insécurité alimentaire des ménages au Canada, Les diététistes du Canada demandent qu'une stratégie pancanadienne chapeautée par le gouvernement soit mise en place pour réduire directement l'insécurité alimentaire au niveau des ménages, y compris des politiques qui s'attaquent aux défis uniques propres à l'insécurité alimentaire dans les ménages des peuples indigènes. Une surveillance régulière de la prévalence et de la gravité de l'insécurité alimentaire des ménages est requise à l'échelle du Canada. Par ailleurs, la recherche doit continuer d'aborder les lacunes en matière de connaissances au sujet de la vulnérabilité des ménages à l'insécurité alimentaire et d'évaluer l'impact des politiques mises au point pour éliminer l'insécurité alimentaire des ménages au Canada.

\section{Les diététistes du Canada recommandent ce qui suit :}

1. L'élaboration et la mise en œuvre d'une stratégie pancanadienne chapeautée par le gouvernement comprenant des politiques et des programmes coordonnés afin de veiller à ce que tous les ménages disposent d'un revenu suffisant et régulier leur permettant de payer les nécessités de base, entre autres la nourriture.

2. La mise en œuvre d'une stratégie soutenue par le gouvernement fédéral visant à aborder de manière complète les défis supplémentaires et uniques propres à l'insécurité alimentaire dans les ménages des peuples indigènes, y compris une garantie de souveraineté alimentaire, avec un accès à des terres et des ressources, pour l'acquisition d'aliments régionaux/traditionnels, de même qu'un accès amélioré à des aliments plus abordables et sains dans les épiceries situées dans les réserves des Premières Nations et les communautés nordiques/éloignées.

3. Un engagement envers la communication et la surveillance obligatoires et annuelles de la prévalence marginale, modérée et grave de l'insécurité alimentaire des ménages dans chaque province et territoire du Canada, y compris au sein des populations vulnérables, de même qu'une évaluation régulière de l'impact de la réduction de la pauvreté et des protocoles de dépistage dans le système de soins de santé.

4. Du soutien pour mener davantage de recherche en vue d'aborder les lacunes en matière de connaissances au sujet des populations où la prévalence et la gravité de l'insécurité alimentaire des ménages sont les plus élevées, et pour orienter la mise en œuvre et l'évaluation de stratégies et de politiques qui élimineront l'insécurité alimentaire des ménages au Canada.

(Rev can prat rech diétét. 2016;77:159)

(DOI: 10.3148/cjdpr-2016-019)

Publié au dcjournal.ca le 19 août 2016.
Access the Household Food Insecurity Background Paper, Position Statement and Recommendations, and Executive Summary, at http://www.dietitians.ca/foodinsecurity.
Vous pouvez accéder au document intitulé L'insécurité alimentaire des ménages au Canada, qui comprend un document d'information, un énoncé de position accompagné de recommandations et un sommaire au http://www.dietetistes. ca/insecuritealimentaire. 